\title{
PROGRAMA DE REABILITAÇÃO RESPIRATÓRIA NA PESSOA SUBMETIDA A CIRURGIA
}

\author{
Joana Maria Pinto Galvão ${ }^{1}$
}

RESUMO: Objetivos: Identificar a evidência científica produzida, relativamente à eficácia dos programas de reabilitação respiratória, nos períodos pré e pós-operatórios, para prevenção da ocorrência de complicações pulmonares pós-operatórias, bem como identificar quais os exercícios respiratórios a incluir.Método: Realizou-se investigação de cariz exploratório, cuja abordagem se baseou em pesquisa bibliográfica nas bases de dados B-ON, EBSCOHOST e plataforma Google Scholar, considerando o espaço temporal de Janeiro de 2017 a Dezembro de 2021, em que os descritores foram conjugados com o boleano "and". Foi norteada pela questão de pesquisa: "Os programas de Reabilitação Respiratória, implementados nos períodos pré e pós-operatórios à pessoa submetida a cirurgia, são efetivos na prevenção de ocorrência de complicações pulmonares pósoperatórias?"Resultados: Do universo de 164 artigos encontrados foram selecionados 8 após aplicação dos critérios de inclusão e exclusão.Conclusão: A realização da presente investigação, demonstrou a eficácia da implementação de programas de RR nos períodos pré e pós-operatórios, na redução da ocorrência de complicações pós-operatórias do foro pulmonar.

Palavras-chave: Exercício Respiratório. Complicações Pós-Operatórias. Período Pré-Operatório. Período Pós-Operatório.

ABSTRACT: Objectives: To identify the scientific evidence produced, regarding the effectiveness of respiratory rehabilitation programs, in the pre and postoperative periods, to prevent the occurrence of postoperative pulmonary complications, as well as to identify which breathing exercises to include.Method: An exploratory investigation was carried out, whose approach was carried out, whose approach was based on bibliographic research in the B-ON, EBSCOHOST and Google Scholar databases, considering the time frame from January 2017 to December 202I, in wich the descriptors were combined with the bolean "and". It was guided by the research question: "Are pulmonary rehabilitation programs, implemented in the pre and postoperative periods for the person undergoing surgery, effective in preventing the occurrence of postoperative pulmonary complications?"Results: From the universe of 164 articles found, 8 were selected after applying the inclusion and exclusion criteria.Conclusion: The present investigation demonstrated the effectiveness of implementing pulmonary rehabilitation programs in the pre and postoperative periods, in reducing the occurrence of postoperative pulmonary complications.

Keywords: Breathing Exercises. Postoperative Complications. Preoperative Period. Postoperative Period.

RESUMEN: Objetivos: Identificar las evidencias científicas producidas sobre la efectividad de los programas de rehabilitación respiratória, en el pre y posoperatorio, para prevenir la ocurrencia de complicaciones pulmonares posoperatorias, así como identificar qué ejercicios respiratórios incluir.Metodo: Se realizó una investigación exploratória, cuyo enfoque se basó en la búsqueda bibliográfica en las bases de datos B-ON, EBSCOHOST y Google Scholar, considerando el marco

${ }^{\mathrm{I}} \mathrm{RN}$, Enfermeira na ULSBA - Hospital José Joaquim Fernandes -Beja. Mestranda em enfermagem e especialidade em Reabilitação ORCID: https://orcid.org/oooo-ooo3-2288-3169 jmp.galvao@gmail.com. 
temporal de Enero de 2017 a Diciembre 2021, en la que se combinaron los descriptores com el boleano "Y". Se guió por la pergunta de investigación: "Los programas de Rehabilitación Respiratoria, implementados en el pre y postoperatorio de la persona intervenida, son efectivos en la prevención de complicaciones pulmonares postoperatorias?"Resultados: Del universo de i64 artículos encontrados, 8 fueron seleccionados después de aplicar los critérios de inclusión y exclusión.Conclusion: La presente investigación demonstró la efectividad de la implementación de programas de rehabilitación respiratória en los períodos pre y postoperatorio, en la reducción de la ocurrencia de complicaciones postoperatorias del foro pulmonar.

Palbaras clave: Ejercicios Respiratorios. Complicaciones Postoperatorias. Periodo Preoperatorio. Periodo Postoperatorio.

\section{ENQUADRAMENTO TEÓRICO}

$\mathrm{O}$ ato cirúrgico constitui-se como abordagem a inúmeros desvios de saúde, no entanto acarreta a possibilidade da ocorrência de complicações como resultado da sua prática.

De entre as complicações pós-operatórias mais frequentes, as complicações do foro pulmonar assumem uma prevalência entre 1\% a 23\% (Miskovic \& Lumb, 2017; Odor et al., 2020). Caracterizam-se como disfunções não intencionais na função pulmonar no período pós-operatório, que influenciam a recuperação da pessoa submetida a cirurgia e são mais

comuns em cirurgias aos segmentos torácico e abdominal (Liu et al., 2020; Lohiya et al., 2018; Lumb, 2019; Schwartz et al., 2020). Mais frequentemente manifestam-se por hipoxemia, atelectasia, pneumonia, broncospasmo, derrame pleural, embolia pulmonar, edema pulmonar, pneumotórax, falência respiratória e infeções (Ávila \& Fenili, 2017; Kendall et al., 2018; Schwartz et al., 2020).

A origem das complicações pós-operatórias do foro pulmonar é multifatorial. Identificam-se na sua génese fatores relacionados com o procedimento cirúrgico, como a indução anestésica, o possível envolvimento do nervo frénico decorrente do segmento anatómico intervencionado e os fenómenos decorrentes do ato cirúrgico, como dor, sedação e imobilidade (Marseu \& Slinger, 2017; Ordem dos Enfermeiros, 2018). Estes fatores, conduzem a diminuição dos volumes pulmonares, verificando-se diminuição da capacidade residual funcional e, portanto, conduzindo a comprometimento da ventilação e do grau de tolerância à atividade, ao que se associa o comprometimento na manutenção da permeabilidade da via aérea, pois o ato de mobilizar secreções fica comprometido pela disfunção do músculo respiratório e pela imobilidade secundária à dor manifestada (Ge et al., 2018). 
Além do procedimento cirúrgico per si, também fatores de risco relacionados com o indivíduo como idade avançada, estado nutricional comprometido, presença de comorbidades e consumo de substâncias nocivas, estão associados a maior risco de desenvolvimento de complicações pulmonares pós-operatórias.

$\mathrm{Na}$ sequência do referido, a implementação de ações com o objetivo de minimizar ou evitar o risco de ocorrência de complicações pulmonares no período pós-operatório assume-se como prioridade. Implementar técnicas de reabilitação respiratória (RR) permite atuar na prevenção de complicações decorrente do procedimento cirúrgico.

A RR é definida como "uma abordagem individualizada e multidisciplinar às pessoas com doença pulmonar crónica, através da aplicação de técnicas e exercícios físicos e respiratórios, e de educação para a saúde, com o objetivo de reduzir a sintomatologia, otimizar o status funcional e melhorar a qualidade de vida" (Spruit, 2014).

$O$ programa de $R R$ inicia-se no período pré-operatório e tem continuidade no período pós-operatório. No período pré-operatório visa a prevenção da ocorrência de complicações pulmonares pós-operatórias, manutenção da função pulmonar, melhorar a taxa de pico de consumo de oxigénio, o volume expiratório forçado e redução do tempo de hospitalização (Fujimoto \& Nakayama, 2019).

O ensino e treino de exercícios respiratórios, no âmbito do programa de RR, vai capacitar o indivíduo submetido a cirurgia a executá-los no pós-operatório imediato, contribuindo assim para minimizar a ocorrência de complicações pulmonares pósoperatórias, aumento da capacidade funcional residual e da qualidade de vida, assim como contribuir para evitar reinternamento hospitalar (Boden et al., 2018; Fujimoto \& Nakayama, 2019).

Atendendo às suas competências específicas, o enfermeiro especialista em enfermagem de reabilitação (EEER) assume-se como um agente ativo no acompanhamento peri operatório da pessoa submetida a cirurgia. Neste sentido é função do EEER assistir no processo de recuperação do paciente com vista à sua máxima capacidade funcional, mantendo a sua auto-estima, qualidade de vida, contribuindo para o seu desenvolvimento pessoal (Diário da República, 2019).

$\mathrm{Na}$ abordagem à pessoa submetida a cirurgia o EEER vai atuar através do diagnóstico precoce e implementação de ações preventivas, neste contexto através da utilização de técnicas de $\mathrm{RR}$, com o objetivo de assegurar a manutenção das capacidades 
funcionais das pessoas após intervenção cirúrgica, prevenindo complicações e maximizando a funcionalidade.

Tendo em conta o descrito, proceder-se-á a uma pesquisa de caráter exploratório com recurso a pesquisa bibliográfica, para identificar a evidência científica produzida relativamente à eficácia dos programas de reabilitação respiratória, nos períodos pré e pósoperatórios, para prevenção da ocorrência de complicações pulmonares pós-operatórias, bem como identificar quais os exercícios respiratórios a incluir.

\section{METODOLOGIA}

No sentido de atingir os objetivos definidos procedeu-se a um desenho de investigação exploratória, cuja abordagem se baseou em pesquisa bibliográfica de modo a obter maior familiaridade com a temática reabilitação respiratória na pessoa submetida cirurgia.

A opção pelo encetar de uma investigação de cariz exploratório assenta no facto de esta metodologia proporcionar maior familiaridade com o problema, tendo em vista tornálo mais explicito (Gil, 2oro). Enquanto instrumento de pesquisa procedeu-se a pesquisa bibliográfica, com base em material publicado, por ter a vantagem de permitir uma cobertura ampla dos fenómenos estudados (Gil, 2010).

O desenvolvimento da presente pesquisa de caráter exploratório, foi norteada pela questão “Os programas de Reabilitação Respiratória, implementados nos períodos pré e pós-operatórios à pessoa submetida a cirurgia, são efetivos na prevenção de ocorrência de complicações pulmonares pós-operatórias?”. A sua elaboração assentou em cinco fases, nomeadamente: I- Delimitação do campo de pesquisa, 2- Definição da Amostra pelo estabelecimento de critérios de inclusão/exclusão dos estudos; 3- Colheita de dados; 4Organização dos Dados; 5- Análise e interpretação dos resultados.

Considerando a questão de investigação, bem como os objetivos traçados, procedeuse no período de 3 de Setembro a 15 de Outubro de 2021, a pesquisa bibliográfica nas bases de dados B-ON e EBSCOHOST através da seleção das bases adicionais CINAHL PLUS with Full Text, Nursing and Allied Health Collection, Cohcrane Plus Collection, MedicLatina e Medline with Full Text.

Para refinar a pesquisa nas bases de dados mencionadas foram utilizados os descritores em ciências da Saúde (DECS) e medical subject heading (MESH) "Breathing 
Exercises", "Postoperative complications", "Preoperative Period", "Postoperative Period", conjugados com boleano "And".

Foram ainda estabelecidos critérios de inclusão e exclusão para triagem dos artigos encontrados. Assim como critérios de inclusão estabeleceu-se: estudos que incluíssem participantes submetidos a cirurgia programada dos segmentos abdominal e torácico e com idade igual ou superior a 18 anos, com intervenção de reabilitação respiratórias nos períodos pré e pós-operatório. Foram considerados todos os tipos de estudos (primários e secundários) disponíveis em full-text e PDF, com autores da área da saúde e revistos por pares, considerando os idiomas inglês, francês e espanhol. Foi estabelecido como espaço temporal para inclusão de artigos o período de oI/oI/2017 a 21/12/202I. Os critérios de exclusão definidos remetem para estudos relacionados com reabilitação respiratória em pessoas submetidas a cirurgia cardíaca ou noutros segmentos que não o torácico e abdominal, bem como artigos publicados em formato de tese, dissertação ou monografia.

Para além do recurso às bases de dados referidas, foi realizada concomitantemente uma pesquisa livre recorrendo à plataforma Google Scholar, utilizando os descritores acima referidos com a mesma conjugação de boleanos.

Face às referências obtidas, procedeu-se a análise das mesmas, mediante leitura crítica e reflexiva dos títulos e resumo, para apurar corresponderem aos critérios de inclusão e exclusão estabelecidos, bem como a adequação à questão de investigação.

Para sistematizar a extração dos dados foi considerado instrumento de colheita de dados que prevê informações acerca de autores, ano de publicação, periódico, base de dados, objetivo do estudo e resultados.

Após análise das referências obtidas, obteve-se uma amostra final de oito artigos que foram integrados para desenvolver a presente pesquisa, cujo processo de seleção, figura no diagrama que se segue 
Revista Ibero- Americana de Humanidades, Ciências e Educação- REASE ${ }^{\text {open }} \mathbf{C}^{\text {access }}$
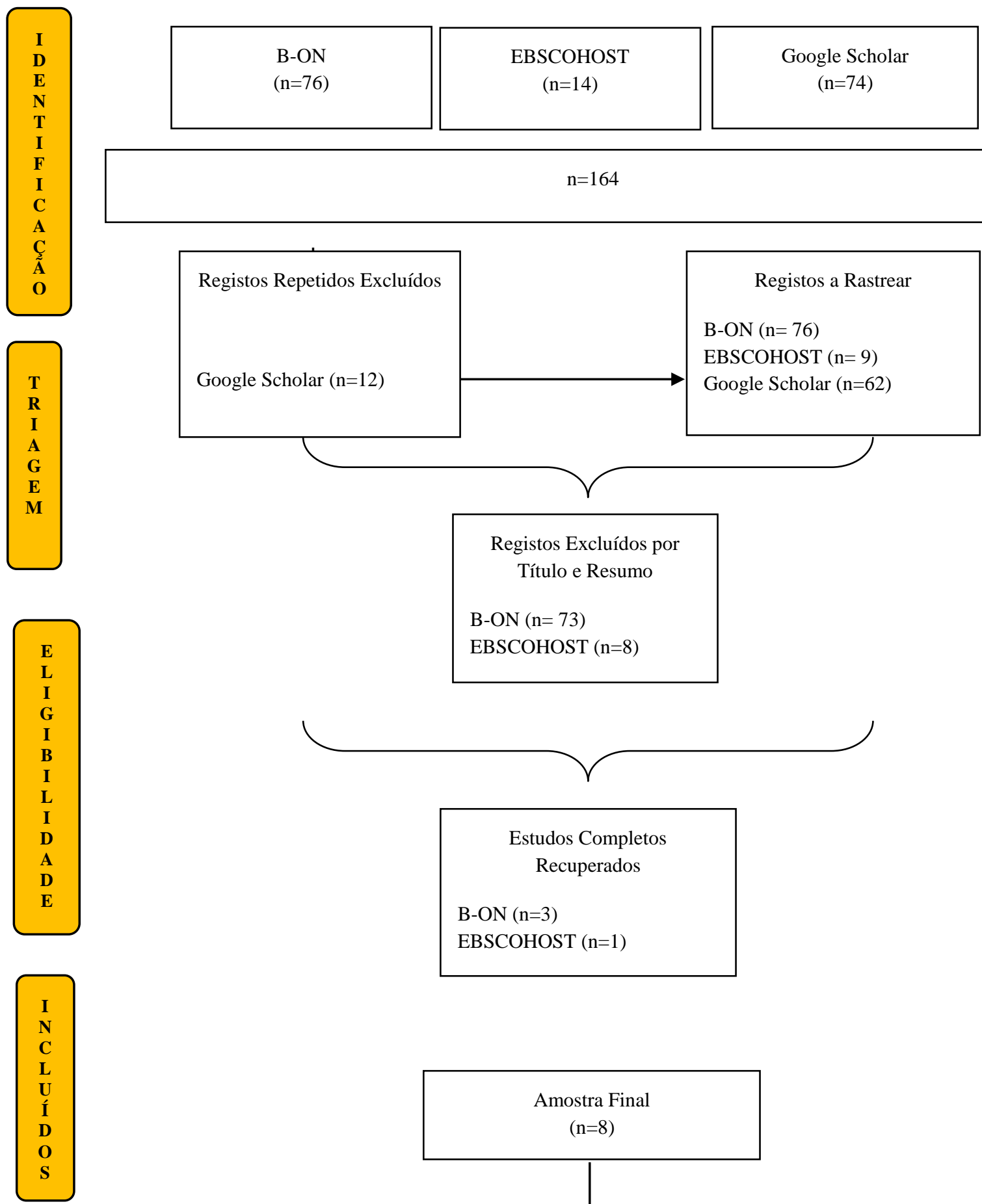

$n=164$
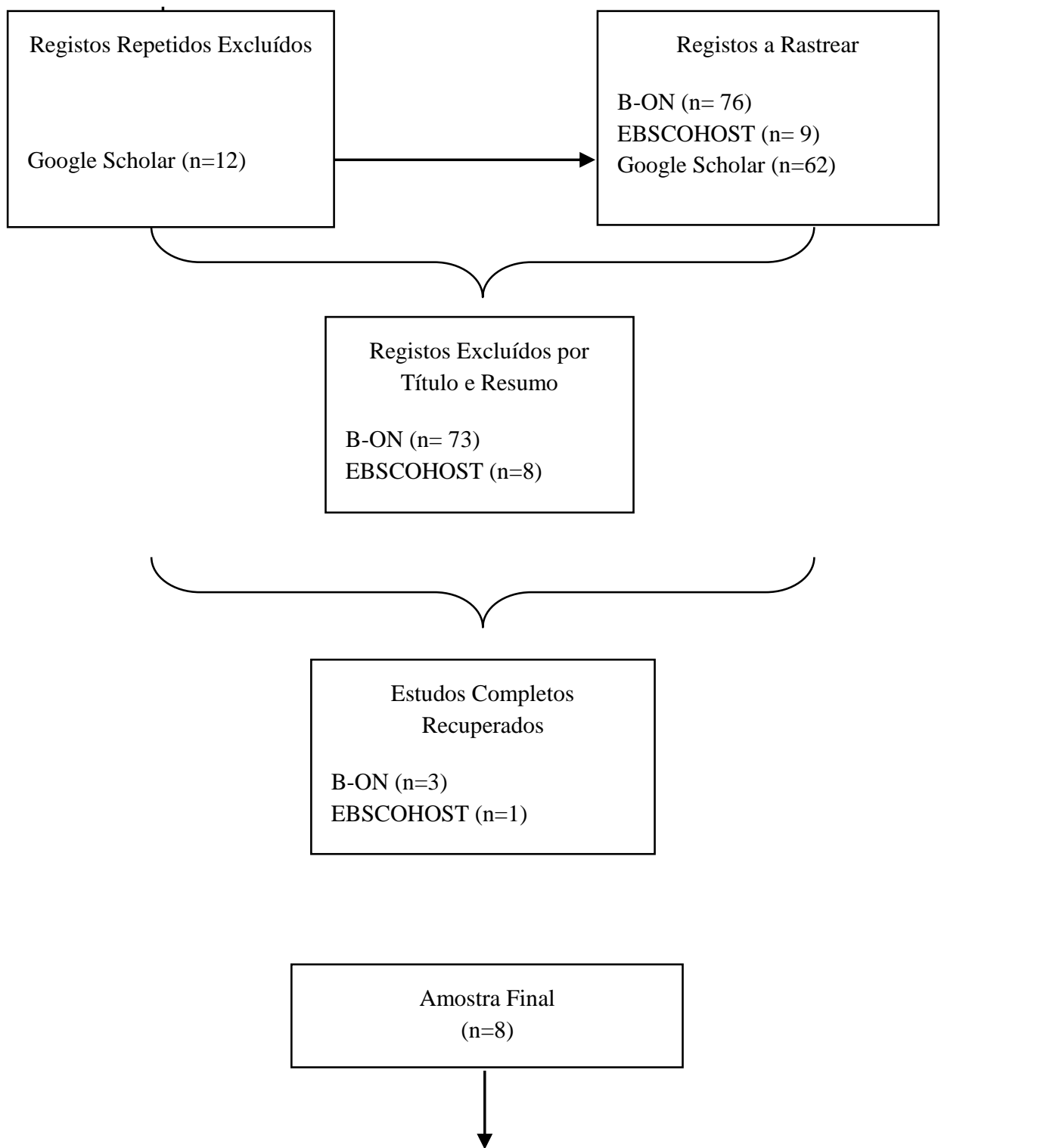

\section{Colheita e organização de dados}

A análise das referências obtidas resultou numa amostra final de oito artigos considerados para desenvolvimento da presente pesquisa. Da leitura e análise destes, procedeu-se a extração de dados, que possibilitou reunir e sintetizar as evidências disponíveis quanto à temática em estudo.

A metodologia de extração de dados, figura na tabela que se segue. 


\begin{tabular}{|c|c|c|c|c|}
\hline $\begin{array}{c}\text { Título/Autor } \\
\text { /Ano }\end{array}$ & $\begin{array}{c}\text { Periódico/Base } \\
\text { de Dados }\end{array}$ & Objetivo & Metodologia & Resultados \\
\hline $\begin{array}{l}\text { "Comprehensive } \\
\text { pulmonary } \\
\text { rehabilitation is an } \\
\text { effective way for } \\
\text { better } \\
\text { postoperative } \\
\text { outcomes in } \\
\text { surgical lung } \\
\text { cancer patients } \\
\text { with Risk Factors: } \\
\text { A propensity } \\
\text { score-Matched } \\
\text { retrospective } \\
\text { cohort study" } \\
\quad \text { (Zhou et al., } \\
\text { 202o) }\end{array}$ & $\begin{array}{c}\text { Cancer } \\
\text { Management and } \\
\text { Research } \\
\text { (Google Scholar) }\end{array}$ & $\begin{array}{c}\text { Investigar a } \\
\text { eficácia e redução } \\
\text { de custos, } \\
\text { resultante da } \\
\text { implementação } \\
\text { de programa de } \\
\text { RR a pessoas com } \\
\text { cancro de pulmão } \\
\text { submetidas a } \\
\text { cirurgia }\end{array}$ & $\begin{array}{l}\text { Estudo de coorte retrospetivo, baseado em } \\
\text { registos clínicos de pessoas com } \\
\text { diagnóstico de cancro do pulmão e alvo de } \\
\text { intervenção cirúrgica, submetidas ou não } \\
\text { a programa de RR. } \\
\text { Para integrar a amostra foram } \\
\text { consideradas pessoas com idade superior a } \\
\text { 7o anos, com previsão de lobectomia sob } \\
\text { toracotomia ou toracoscopia vídeo- } \\
\text { assistida e antecedentes de doença } \\
\text { pulmonar obstrutiva crónica (DPOC), } \\
\text { hábitos tabágicos e previsão de FEVi } \\
\text { inferior a 6o\% no pós-operatório. Foram } \\
\text { excluídos indivíduos que recusaram } \\
\text { participar ou com condições adversas } \\
\text { como acidente vascular cerebral, } \\
\text { hemoptises, arritmia ou distúrbio } \\
\text { músculo-esquelético. Os participantes que } \\
\text { integraram o grupo de intervenção tinham } \\
\text { de ser admitidos } 7 \text { dias antes da cirurgia. } \\
\text { O programa de intervenção incluía treino } \\
\text { da musculatura respiratória com recurso a } \\
\text { inspirómetro de incentivo fluxo- } \\
\text { dependente, respiração abdomino- } \\
\text { diafragmática e treino aeróbio. }\end{array}$ & $\begin{array}{c}\text { Verificou-se } \\
\text { menor incidência } \\
\text { de complicações } \\
\text { pós-operatórias } \\
\text { do foro } \\
\text { pulmonar, bem } \\
\text { como menor } \\
\text { tempo de } \\
\text { internamento } \\
\text { hospitalar e } \\
\text { consequente } \\
\text { diminuição dos } \\
\text { gastos em saúde, } \\
\text { nos participantes } \\
\text { que integraram o } \\
\text { grupo de } \\
\text { intervenção. }\end{array}$ \\
\hline $\begin{array}{l}\text { "Effects of } \\
\text { respiratory } \\
\text { rehabilitation } \\
\text { nursing on } \\
\text { improving } \\
\text { postoperative } \\
\text { respiratory } \\
\text { function and } \\
\text { quality of life of } \\
\text { patients with lung } \\
\text { cancer surgery" } \\
\text { (Liu et al., 2020) }\end{array}$ & $\begin{array}{l}\text { International } \\
\text { Journal of Clinical } \\
\text { and Experimental } \\
\text { Medicine } \\
\text { (Google Scholar) }\end{array}$ & $\begin{array}{c}\text { Explorar os } \\
\text { efeitos de } \\
\text { programas de RR, } \\
\text { implementados } \\
\text { por EEER, na } \\
\text { função } \\
\text { respiratória e } \\
\text { qualidade de } \\
\text { vida, no período } \\
\text { pós-operatório da } \\
\text { pessoa submetida } \\
\text { a cirurgia no } \\
\text { âmbito de cancro } \\
\text { do pulmão. }\end{array}$ & $\begin{array}{l}\text { Ensaio de controlo randomizado, com } \\
\text { uma amostra de io6 participantes, com } \\
\text { idades compreendidas entre } 55 \text { e } 75 \text { anos e } \\
\text { com diagnóstico de cancro do pulmão, } \\
\text { submetidos a cirurgia. } \\
\text { Os participantes foram aleatoriamente } \\
\text { divididos em grupo de controlo e } \\
\text { intervenção. Os participantes do grupo de } \\
\text { intervenção receberam cuidados } \\
\text { diferenciados por parte de EEER, que } \\
\text { incluíram exercícios de respiração } \\
\text { abdomino-diafragmática, expiração com } \\
\text { lábios semicerrados, tosse dirigida e } \\
\text { exercícios respiratórios resistidos com uso } \\
\text { de balão. Os participantes do grupo de } \\
\text { controlo receberam cuidados gerais de } \\
\text { enfermagem. }\end{array}$ & $\begin{array}{l}\text { A implementação } \\
\text { do programa de } \\
\text { RR por EEER em } \\
\text { pessoas com } \\
\text { cancro de pulmão } \\
\text { submetidas a } \\
\text { intervenção } \\
\text { cirúrgica, } \\
\text { demonstrou ser } \\
\text { eficaz na } \\
\text { maximização da } \\
\text { função } \\
\text { respiratória, } \\
\text { diminuindo } \\
\text { assim a } \\
\text { incidência de } \\
\text { complicações } \\
\text { pós-operatórias } \\
\text { de foro pulmonar } \\
\text { e } \\
\text { consequentement } \\
\text { e melhorou a } \\
\text { habilidade destes } \\
\text { para o } \\
\text { autocuidado }\end{array}$ \\
\hline $\begin{array}{l}\text { "Effect of Chest } \\
\text { Physiotherapy } \\
\text { along with early } \\
\text { mobilization after } \\
\text { abdominal } \\
\text { surgery" }\end{array}$ & $\begin{array}{l}\text { European Journal } \\
\text { of Medical and } \\
\text { Health Sciences } \\
\text { (Google Scholar) }\end{array}$ & $\begin{array}{l}\text { Avaliar o efeito } \\
\text { da fisioterapia } \\
\text { respiratória a solo } \\
\text { e associada a } \\
\text { mobilização } \\
\text { precoce, em } \\
\text { pessoas }\end{array}$ & $\begin{array}{l}\text { Estudo quase experimental, com } 60 \\
\text { participantes submetidos a cirurgia } \\
\text { abdominal alta sob anestesia geral, com } \\
\text { idade superior a is anos e inferior a 7o } \\
\text { anos, que acordassem em participar. } \\
\text { Foram excluídos participantes que não }\end{array}$ & $\begin{array}{c}\text { Verificou-se } \\
\text { maior eficácia da } \\
\text { fisioterapia } \\
\text { respiratória } \\
\text { associada a } \\
\text { mobilização } \\
\text { precoce, }\end{array}$ \\
\hline
\end{tabular}




\begin{tabular}{|c|c|c|c|c|}
\hline $\begin{array}{c}\text { (Kabir et al., } \\
\text { 202I) }\end{array}$ & & $\begin{array}{c}\text { submetidas a } \\
\text { cirurgia } \\
\text { abdominal de } \\
\text { grande porte, em } \\
\text { variáveis como } \\
\text { tempo de } \\
\text { internamento } \\
\text { hospitalar, } \\
\text { intensidade da } \\
\text { dor, nível de } \\
\text { saturação de } \\
\text { oxigénio e nível } \\
\text { de independência } \\
\text { funcional. }\end{array}$ & $\begin{array}{l}\text { correspondessem à faixa etária ou tipo de } \\
\text { anestesia definidas para inclusão, com } \\
\text { compromisso neuromuscular, submetidos } \\
\text { a cirurgia sob técnica de laparoscopia } \\
\text { orientada por vídeo e antecedente de } \\
\text { DPOC. } \\
\text { Os participantes foram divididos } \\
\text { aleatoriamente em grupo de controlo, que } \\
\text { recebeu cuidados pós-operatórios de } \\
\text { rotina, e grupo de intervenção, que } \\
\text { integrou programa de fisioterapia } \\
\text { respiratória que incluiu exercícios de } \\
\text { respiração profunda seguidos de tosse ou } \\
\text { Huff e mobilização precoce. } \\
\text { Para avaliação das variáveis recorreu-se a } \\
\text { avaliação de saturação periférica de } \\
\text { oxigénio (SPOz) pelo uso de oxímetro, } \\
\text { escala de esforço modificada de Borg, } \\
\text { escala numérica da dor e Medida de } \\
\text { Independência Funcional. }\end{array}$ & $\begin{array}{c}\text { comparativament } \\
\text { e à } \\
\text { implementação } \\
\text { de fisioterapia } \\
\text { respiratória } \\
\text { unicamente. } \\
\text { Os participantes } \\
\text { que receberam } \\
\text { combinação de } \\
\text { fisioterapia } \\
\text { respiratória e } \\
\text { mobilização } \\
\text { precoce tiveram } \\
\text { menor tempo de } \\
\text { internamento } \\
\text { hospitalar, maior } \\
\text { nível de } \\
\text { saturação } \\
\text { periférica de } \\
\text { oxigénio e maior } \\
\text { capacidade } \\
\text { funcional para o } \\
\text { autocuidado. }\end{array}$ \\
\hline $\begin{array}{l}\text { "Clinical } \\
\text { guidelines on } \\
\text { perioperative } \\
\text { management } \\
\text { strategies for } \\
\text { enhanced recovery } \\
\text { after lung surgery" } \\
\text { (Gao et al., 2019) }\end{array}$ & $\begin{array}{l}\text { Translational Lung } \\
\text { Cancer Research } \\
\text { (Google Scholar) }\end{array}$ & $\begin{array}{c}\text { Estabelecer } \\
\text { diretrizes clínicas } \\
\text { para manutenção } \\
\text { da função } \\
\text { respiratória, no } \\
\text { período } \\
\text { perioperatório, da } \\
\text { pessoa submetida } \\
\text { a cirurgia } \\
\text { pulmonar. } \\
\text { Rever medidas } \\
\text { perioperatórias } \\
\text { baseadas no } \\
\text { programa } \\
\text { “Enhaced } \\
\text { Recovery after } \\
\text { Surgery (ERAS) } \\
\text { para cirurgia } \\
\text { torácica }\end{array}$ & $\begin{array}{l}\text { Foram desenvolvidas diretrizes clínicas, } \\
\text { após revisão crítica da literatura relativa a } \\
\text { gestão da função respiratória da pessoa } \\
\text { submetida a cirurgia pulmonar. } \\
\text { As orientações desenvolvidas incluem } \\
\text { preparação pré-operatória (educação para } \\
\text { a saúde, cessação tabágica, avaliação da } \\
\text { função pulmonar pré-operatória, gestão de } \\
\text { vias aéreas e tratamento anti-infecioso } \\
\text { profilático), intraoperatória (anestesia e } \\
\text { tratamento cirúrgico) e pós-operatória } \\
\text { (gestão pós-operatória das vias aéreas, } \\
\text { reabilitação pulmonar, deambulação } \\
\text { precoce e uso de analgesia). Foi também } \\
\text { considerada a opinião de peritos, quanto a } \\
\text { estas temáticas. }\end{array}$ & $\begin{array}{l}\text { Espera-se que as } \\
\text { diretrizes clínicas } \\
\text { desenvolvidas, } \\
\text { acerca da gestão } \\
\text { perioperatória da } \\
\text { pessoa submetida } \\
\text { a cirurgia } \\
\text { pulmonar, } \\
\text { permitam colocar } \\
\text { o conhecimento } \\
\text { existente ao } \\
\text { dispor da prática } \\
\text { clínica. }\end{array}$ \\
\hline $\begin{array}{l}\text { "Essentials of } \\
\text { physiotherapy } \\
\text { after thoracic } \\
\text { surgery: What } \\
\text { physiotherapists } \\
\text { need to know, a } \\
\text { narrative review" } \\
\text { (Ahmad, 2018) }\end{array}$ & $\begin{array}{c}\text { The Korean } \\
\text { Journal of Thoracic } \\
\text { and Cardiovascular } \\
\text { Surgery } \\
\text { (B-ON) }\end{array}$ & $\begin{array}{l}\text { Apresentar os } \\
\text { princípios } \\
\text { orientadores para } \\
\text { implementação } \\
\text { de programa de } \\
\text { fisioterapia } \\
\text { respiratória, a } \\
\text { pessoas } \\
\text { submetidas a } \\
\text { cirurgia torácica }\end{array}$ & $\begin{array}{l}\text { Revisão narrativa de literatura, com o } \\
\text { intuito de orientar a prática clínica do } \\
\text { profissional de fisioterapia, no cuidado } \\
\text { pós-operatório à pessoa submetida a } \\
\text { cirurgia torácica. }\end{array}$ & $\begin{array}{l}\text { Compilação dos } \\
\text { princípios de } \\
\text { fisioterapia a } \\
\text { implementar, } \\
\text { após cirurgia } \\
\text { torácica, } \\
\text { juntamente com } \\
\text { uma descrição } \\
\text { detalhada das } \\
\text { intervenções, } \\
\text { para proporcionar } \\
\text { orientação clínica }\end{array}$ \\
\hline
\end{tabular}




\begin{tabular}{|c|c|c|c|c|}
\hline $\begin{array}{l}\text { breathing exercises } \\
\text { in subjects with } \\
\text { lung cancer } \\
\text { undergoing } \\
\text { surgical resection: } \\
\text { A systematic } \\
\text { review and meta- } \\
\text { analysis" } \\
\text { (Wang et al., } \\
\text { 2019) }\end{array}$ & $\begin{array}{l}\text { Nursing } \\
(\mathrm{B}-\mathrm{ON})\end{array}$ & $\begin{array}{c}\text { da } \\
\text { implementação } \\
\text { de exercícios } \\
\text { respiratórios no } \\
\text { período pós- } \\
\text { operatório, na } \\
\text { função pulmonar, } \\
\text { distância } \\
\text { percorrida, tempo } \\
\text { de internamento } \\
\text { e incidência de } \\
\text { ocorrência de } \\
\text { complicações } \\
\text { pós-operatórias } \\
\text { do foro } \\
\text { pulmonar, } \\
\text { considerando a } \\
\text { pessoa submetida } \\
\text { a cirurgia, no } \\
\text { âmbito de cancro } \\
\text { do pulmão }\end{array}$ & $\begin{array}{l}\text { análise, com recurso às bases de dados } \\
\text { Pubmed, Embase Ovid, Cochrane Central } \\
\text { Register of Randomized, Web of science e } \\
\text { Wanfang Weipu. Foram considerados } \\
\text { exclusivamente artigos com metodologia } \\
\text { de ensaio clínico randomizado que } \\
\text { incluíssem pessoas com cancro do pulmão, } \\
\text { com necessidade de cirurgia e submetidas } \\
\text { a RR no período perioperatório. Foram } \\
\text { considerados I6 artigos, em que o risco de } \\
\text { enviesamento foi avaliado com recurso ao } \\
\text { instrumento "Cocrhane Colaboration risk } \\
\text { of bias" }\end{array}$ & $\begin{array}{l}\text { mostra que os } \\
\text { exercícios } \\
\text { respiratórios } \\
\text { podem melhorar } \\
\text { a função } \\
\text { pulmonar, } \\
\text { diminuir a } \\
\text { incidência de } \\
\text { complicações } \\
\text { pós-operatórias } \\
\text { pulmonares, bem } \\
\text { como o tempo de } \\
\text { internamento em } \\
\text { pessoas com } \\
\text { cancro do pulmão } \\
\text { submetidas a } \\
\text { cirurgia } \\
\text { pulmonar }\end{array}$ \\
\hline $\begin{array}{l}\text { "Effect of } \\
\text { combination of pre } \\
\text { and postoperative } \\
\text { pulmonary } \\
\text { rehabilitation on } \\
\text { onset of } \\
\text { postoperative } \\
\text { pneumonia: a } \\
\text { retrospective } \\
\text { cohort study based } \\
\text { on data from the } \\
\text { diagnosis } \\
\text { procedure } \\
\text { combination } \\
\text { database in Japan } \\
\text { (Fujimoto \& } \\
\text { Nakayama, 2or9) }\end{array}$ & $\begin{array}{c}\text { International } \\
\text { Journal of Clinical } \\
\text { Oncology } \\
\text { (B-ON) }\end{array}$ & $\begin{array}{l}\text { Verificar o efeito } \\
\text { da RR, na } \\
\text { incidência de } \\
\text { pneumonia pós- } \\
\text { operatória, } \\
\text { quando realizada } \\
\text { em combinação } \\
\text { antes e após } \\
\text { cirurgia } \\
\text { pulmonar, } \\
\text { comparativament } \\
\text { e com } \\
\text { intervenções de } \\
\text { RR realizadas } \\
\text { unicamente num } \\
\text { destes períodos e } \\
\text { cm situações em } \\
\text { que não decorreu } \\
\text { intervenção de } \\
\text { RR }\end{array}$ & $\begin{array}{l}\text { Estudo de coorte retrospetivo, em que os } \\
\text { dados foram obtidos a partir da base de } \\
\text { dados clínica "Diagnosis Procedure } \\
\text { Combination". Foram selecionados dados } \\
\text { de pessoas com tumor pulmonar primário, } \\
\text { submetidas a pneumectomia via } \\
\text { toracotomia ou toracoscopia. Foram } \\
\text { excluídos dados de indivíduos que } \\
\text { faleceram na sequência de cancro do } \\
\text { pulmão antes da cirurgia, submetidas a } \\
\text { transplante pulmonar e diagnóstico de } \\
\text { pneumonia. Foram considerados I5, I46 } \\
\text { registos, dos quais houve participantes que } \\
\text { integraram programa de RR nos períodos } \\
\text { pré e pós-operatório, outros que } \\
\text { integraram programa de RR em apenas } \\
\text { um destes períodos e os que não } \\
\text { integraram programa de RR. Para } \\
\text { avaliação das variáveis foi usado The } \\
\text { Hugh Jones Classification para dispneia; o } \\
\text { TNM staging system para definir estadio } \\
\text { do cancro e o índice de Barthel para } \\
\text { avaliar a performance nas atividades de } \\
\text { vida diária. }\end{array}$ & $\begin{array}{c}\text { A implementação } \\
\text { de programas de } \\
\text { RR no período } \\
\text { pré-operatório e } \\
\text { continuados no } \\
\text { pós-operatório } \\
\text { mostra-se mais } \\
\text { eficaz na } \\
\text { prevenção da } \\
\text { ocorrência de } \\
\text { pneumonia pós- } \\
\text { operatória } \\
\text { comparativament } \\
\text { e com programas } \\
\text { de RR } \\
\text { implementados } \\
\text { apenas num } \\
\text { destes períodos } \\
\text { ou em caso de } \\
\text { ausência de } \\
\text { intervenção. }\end{array}$ \\
\hline $\begin{array}{c}\text { "Prophylatic } \\
\text { Chest } \\
\text { Physiotherapy in } \\
\text { Major Abdominal } \\
\text { Surgery among } \\
\text { Elderly Patients" } \\
\text { (Lohiya et al., } \\
\text { 2018) }\end{array}$ & $\begin{array}{l}\text { Journal of The } \\
\text { Indian Academy of } \\
\text { Geriatrics } \\
\text { (EBSCOHOST) }\end{array}$ & $\begin{array}{l}\text { Avaliar o efeito } \\
\text { do recurso a } \\
\text { fisioterapia } \\
\text { respiratória } \\
\text { profilática, na } \\
\text { incidência de } \\
\text { complicações } \\
\text { pulmonares pós- } \\
\text { operatórias após } \\
\text { cirurgia } \\
\text { abdominal. }\end{array}$ & $\begin{array}{l}\text { Ensaio de controlo randomizado, com } \\
\text { uma amostra de so pessoas, com idade } \\
\text { superior a 6o anos, submetidas a cirurgia } \\
\text { abdominal eletiva. } \\
\text { Aleatoriamente os participantes foram } \\
\text { divididos em grupo de controlo e } \\
\text { intervenção. O grupo de intervenção } \\
\text { integrou sessões de educação para a saúde } \\
\text { acerca do período peri-operatório e foi } \\
\text { subdividido em dois subgrupos quanto às } \\
\text { técnicas de fisioterapia respiratória que } \\
\text { foram aplicadas, sendo que os } \\
\text { participantes do subgrupo A realizaram } \\
\text { inspirometria de incentivo, e os do } \\
\text { subgrupo B realizaram exercícios de } \\
\text { respiração profunda com expiração com } \\
\text { lábios semicerrados e tosse dirigida de } \\
\text { hora a hora. }\end{array}$ & $\begin{array}{l}\text { A incidência de } \\
\text { ocorrência de } \\
\text { complicações } \\
\text { pós-operatórias } \\
\text { de foro pulmonar } \\
\text { foi superior no } \\
\text { grupo de } \\
\text { controlo, } \\
\text { comparativament } \\
\text { e com ambos os } \\
\text { subgrupos de } \\
\text { intervenção. } \\
\text { Entre os } \\
\text { subgrupos de } \\
\text { intervenção, a } \\
\text { incidência de } \\
\text { complicações } \\
\text { pós-operatórias }\end{array}$ \\
\hline
\end{tabular}




\begin{tabular}{|l|l|l|c|}
\hline & & $\begin{array}{l}\text { Os participantes do grupo de controlo não } \\
\text { integraram nenhum tipo de intervenção. }\end{array}$ & $\begin{array}{c}\text { pulmonares foi } \\
\text { inferior no } \\
\text { subgrupo que } \\
\text { realizou }\end{array}$ \\
& & $\begin{array}{l}\text { A avaliação decorreu no período pós- } \\
\text { operatório, com recurso a oximetria de } \\
\text { pulso e monitorização do } \\
\text { desenvolvimento de sintomas sugestivos } \\
\text { de alteração da função respiratória. }\end{array}$ & $\begin{array}{c}\text { inspirometria de } \\
\text { incentivo. }\end{array}$ \\
\hline
\end{tabular}

Análise e interpretação dos dados

As complicações pós-operatórias do foro pulmonar constituem-se como principal causa de morbilidade e mortalidade nas pessoas submetidas a cirurgia, sobretudo se esta decorrer nos segmentos torácico e abdominal, acima da linha umbilical (Liu et al., 2020; Lohiya et al., 2018).

A bibliografia considerada, remete para a eficácia da implementação de programas de RR, para prevenir a ocorrência de complicações pós-operatórias do foro pulmonar.

Assim, considerando a questão norteadora da pesquisa e os objetivos traçados, a análise e interpretação de dados visa determinar a eficácia da implementação de programas de $R R$, nos períodos pré e pós-operatórios à pessoa submetida a cirurgia, com o intuito de prevenir a ocorrência de complicações pulmonares pós-operatórias, bem como determinar os exercícios respiratórios a considerar no seu desenvolvimento.

Eficácia da implementação de programa de reabilitação respiratória, nos períodos pré e pós-operatórios à pessoa submetida a cirurgia, na prevenção de complicações pósoperatórias do foro pulmonar

A evidência incluída é unânime em demonstrar a eficácia da implementação de programas de $\mathrm{RR}$, nos períodos pré e pós-operatórios, no que concerne a redução da ocorrência de complicações pós-operatórias do foro pulmonar.

A participação em programa de RR permite maximizar a função pulmonar (Liu et al., 2020), pelo que participantes que integraram programas de $R R$ no período perioperatório, demonstram menor incidência de complicações pulmonares do foro pulmonar e como consequência o tempo internamento em instituição hospitalar para recuperação pós-operatória é menor (Kabir et al., 2021; Liu et al., 2020; Zhou et al., 2020).

Relativamente à implementação do programa de $R R$, fica demonstrado a sua maior efetividade na prevenção da ocorrência de complicações pós-operatórias do foro pulmonar, quando estes são introduzidos concomitantemente nos períodos pré e pós-operatórios comparativamente à sua introdução unicamente num destes períodos, ou quando não são introduzidos em nenhum momento. (Fujimoto \& Nakayama, 2019). 
No planeamento dos programas de $\mathrm{R}$, a literatura considerada, sugere os domínios a incluir, atendendo ao momento perioperatório em que será implementado. Assim no período pré-operatório, o objetivo é possibilitar à pessoa compreender todo o processo cirúrgico e torná-la agente ativo do processo de saúde pela inclusão dos seus objetivos, para tal, o programa de RR assenta na educação para a saúde com enfoque no ato cirúrgico, adoção de estilos de vida saudáveis através de alimentação equilibrada e cessação tabágica e alcoólica e maximização da função pulmonar através de estratégias de reabilitação respiratória (Gao et al., 2019).

Após a cirurgia o enfoque assenta na gestão da função pulmonar, controlo da dor e deambulação precoce (Gao et al., 2019).

$\mathrm{O}$ programa de $\mathrm{RR}$, no período pré-operatório, possibilita aos integrantes melhoria da função pulmonar, maximização da função motora e marcha. Já no que respeita à sua implementação no período pós-operatório, os integrantes demonstram melhoria no parâmetro FEVI, melhoria na qualidade de vida, além de contribuir para melhorar a amplitude de movimentos e maximizar a independência funcional (Fujimoto \& Nakayama, 2019; Kabir et al., 2021).

Em suma, a implementação de programa de reabilitação respiratória a pessoas submetidas a cirurgia, possibilita melhorar a função respiratória, reduzir a incidência de complicações pulmonares pós-operatórias e melhorar a capacidade para o autocuidado e neste sentido, potenciar maior qualidade de vida. Atendendo às suas competências específicas e papel na equipa multiprofissional, o EEER ao implementar programas de reabilitação respiratória no acompanhamento perioperatório da pessoa submetida a cirurgia, possibilita a maximização da sua funcionalidade pelo desenvolvimento das suas capacidades, contribuindo para melhor desempenho respiratório, potenciando assim o seu rendimento e desenvolvimento pessoal (Diário da República, 2019).

\section{EXERCÍCIOS RESPIRATÓRIOS}

A intervenção cirúrgica, atendendo ao tipo e segmento anatómico onde é realizada, conduz a enfraquecimento da musculatura respiratória, pelo que implementar programas de $\mathrm{RR}$ que fomentem o treino $\mathrm{da}$ musculatura respiratória, possibilita às pessoas submetidas a cirurgia melhorar a função respiratória, tolerância ao exercício, bem como reduzir o desconforto respiratório (Wang et al., 2019). Os exercícios respiratórios 
contribuem para melhorar a função dos músculos respiratórios, reduzir a limitação da ventilação nas atividades de vida diária e melhorar a atividade, ao permitirem reduzir a competição pelo aporte de sangue entre músculos respiratórios e periféricos (Wang et al., 2019).

$\mathrm{Na}$ implementação de programa de RR no período pré-operatório, com o objetivo de aumentar a função e capacidade pulmonar, a componente educacional assume um papel de destaque no ensino e treino de exercícios de respiração profunda, tosse, exercícios de expansão pulmonar e treino de musculatura inspiratória. A estes exercícios respiratórios, associa-se treino aeróbico (Fujimoto \& Nakayama, 2019; Gao et al., 2019; Lohiya et al., 2018; Wang et al., 2019; Zhou et al., 2020). Já no período pós-operatório o enfoque assenta na mobilização precoce, limpeza das vias aéreas, correção postural, reeducação respiratória no esforço e continuidade dos exercícios respiratórios iniciados no período préoperatório(Ahmad, 2018; Gao et al., 2019; Kabir et al., 2021; Liu et al., 2020; Wang et al., 2019).

Explorando os exercícios supracitados, a respiração profunda permite melhorar a expansão pulmonar e aumentar o volume e capacidade pulmonar após cirurgia (Ahmad, 2018). É recomendada a sua realização na posição sentado, mas é igualmente possível a sua realização enquanto esta posição não é recomendada. Na sua realização a pessoa é instruída a inspirar pelo nariz e suster a respiração por 2 segundos e seguidamente a expirar pela boca lentamente, com os lábios franzidos. No período pós-operatório a pessoa deve ser instruída a realizar contenção da incisão cirúrgica, aquando da realização deste exercício, afim de minimizar a dor (Ahmad, 2018; Fujimoto \& Nakayama, 2019; Gao et al., 2019; Kabir et al., 2021; Lohiya et al., 2018; Zhou et al., 2020). Estes exercícios podem ainda ser combinados com mobilização de membros superiores e tronco (Ahmad, 2018). A associação entre respiração profunda e a instrução para direcionar a respiração para o diafragma melhora a eficiência da ventilação e promove um padrão respiratório fisiológico. Pode ser realizado na posição sentado ou deitado, em que como estímulo a pessoa é instruída a colocar as mãos na região epigástrica e apical e instruída a respirar para o abdómen e parte inferior do tórax (Gao et al., 2019; Kabir et al., 2021; Liu et al., 2020; Zhou et al., 2020).

O recurso a inspirómetro de incentivo, para treino da musculatura inspiratória, foi abordado em alguns dos estudos considerados, sendo considerado eficaz na prevenção da 
ocorrência de complicações pulmonares pós-operatórias e melhoria da função pulmonar (Gao et al., 2019; Kabir et al., 202ı; Lohiya et al., 2018; Zhou et al., 2020). Para utilização do inspirómetro de incentivo a pessoa é instruída a colocar-se na posição sentado e colocar o dispositivo à altura dos olhos para visualização do incentivo. Posteriormente é instruída a expirar, exalando o máximo de ar dos pulmões, e seguidamente a realizar uma inspiração lenta e profunda através do bocal do inspirómetro (Kabir et al., 2021; Zhou et al., 2020). Apesar da sua inclusão nos programas de exercícios de forma rotineira, a sua eficácia ainda é dúbia, os estudos considerados mostram benefícios quanto à sua utilização no âmbito de cirurgia abdominal, na redução de complicações pulmonares pós-operatórias comparativamente com exercícios de respiração profunda incluídos em exclusivo (Kabir et al., 2021; Lohiya et al., 2018), no entanto no âmbito da cirurgia torácica, não há evidência que a sua utilização seja mais eficaz comparativamente a exercícios de respiração profunda, constituindo-se no entanto como uma ferramenta útil na avaliação da capacidade pulmonar (Ahmad, 2018).

Após a cirurgia, a inabilidade para tossir e promover a limpeza das vias aéreas conduz ao aumento do risco de infeção (Ahmad, 2018). Assim é importante dar início a técnicas de limpeza da via aérea no período pré-operatório e no pós-operatório imediato. Existem várias técnicas para promover a limpeza da via aérea, como tosse assistida, tose dirigida, técnica de expiração forçada, técnica de ciclo ativo da respiração, drenagem postural modificada e pressão expiratória positiva. Os estudos incluídos abordam a utilização da técnica de tosse dirigida ou assistida e Huff (Gao et al., 2019; Kabir et al., 2021; Wang et al., 2019) para promover a limpeza das vias aéreas. No entanto não existe evidência quanto à eficácia de uma técnica de limpeza das vias aéreas sobre outra, dependendo o método a adotar da preferência da pessoa intervencionada (Ahmad, 2018). A execução da técnica de limpeza das vias aéreas pode agravar a dor sobre o local de incisão cirúrgica, pelo que instruir a pessoa a conter a incisão cirúrgica, aquando da realização da técnica de limpeza da via aérea, é recomendada (Ahmad, 2018).

Os estudos incluídos abordam a importância da mobilização precoce no período pós-operatório (Ahmad, 2018; Gao et al., 2019; Kabir et al., 2021; Wang et al., 2019).

No entanto a mobilização tem de ser faseada e ocorrer quando a pessoa submetida a cirurgia apresentar estabilidade hemodinâmica. Primeiramente a pessoa é instruída a sentar-se no leito, se tolerar coloca-se de pé e se tolerar inicia marcha. Durante estas fases 
procede-se a avaliação hemodinâmica (Ahmad, 2018; Kabir et al., 202I). Introduz-se períodos de deambulação de forma gradual na sua duração e atendendo à tolerância da pessoa submetida a cirurgia. $O$ treino de escadas tem início quando a pessoa consegue deambular uma distância considerável numa superfície plana, mantendo estabilidade hemodinâmica (Ahmad, 2018; Kabir et al., 2021).

As pessoas submetidas a cirurgia que não sejam elegíveis para iniciar deambulação podem iniciar exercícios respiratórios, de limpeza da via aérea e cardiovasculares no leito (Ahmad, 2018). Estão indicados exercícios de mobilização passiva, ativa-assistida ou ativa ao nível dos membros inferiores, para prevenir estase circulatória. Mobilizar todos os segmentos dos membros superiores também é de considerar por permitir a mobilidade da caixa torácica e mobilização do tronco através da extensão, flexão lateral e rotação, sempre com contenção da incisão cirúrgica para minimizara dor (Ahmad, 2018).

Mediante a progressão da pessoa ao longo do programa de $\mathrm{R}$, a introdução do treino aeróbio prevê a utilização de resistência, mediante a tolerância da pessoa e de forma gradual. A utilização de dispositivos como bicicleta estática e passadeira pode ser incluído, o tempo de treino é crescente em duração (Gao et al., 2019; Zhou et al., 2020). Aquando da realização de esforço, a pessoa submetida a cirurgia pode ser instruída quanto à forma de respirar no esforço, inspirar em repouso e expirar quando inicia movimento, de modo a proporcionar maior grau de tolerância (Ahmad, 2018).

Importa ainda mencionar, que na sequência da cirurgia, a pessoa pode adotar posturas incorretas, na sequência da dor implícita, pelo que incluir no programa de $R R$ medidas de correção postural é necessário para promover a ventilação, recorrendo a espelho na instrução da pessoa quanto à postura a manter (Ahmad, 2018; Kabir et al., 202I).

Atendendo ao mencionado, os exercícios respiratórios constituem-se como estratégias a ser empregues na reabilitação da pessoa submetida a cirurgia, com vista a recuperação e maximização da sua capacidade funcional e desempenho respiratóri

\section{CONSIDERAÇÕES FINAIS}

Com a realização desta investigação de caráter exploratório, foi possível apurar a eficácia da implementação de programas de $R R$, nos períodos pré e pós-operatórios à pessoa submetida a cirurgia. A literatura demonstrou a sua eficácia na prevenção da 
ocorrência de complicações pós-operatórias do foro pulmonar, por promoverem recuperação e maximização da capacidade e função pulmonar.

A evidência demonstrou que a aplicação dos programas de $R R$, nos períodos pré e pós-operatórios, permite acelerar a recuperação funcional pós-operatória e diminuir o tempo de internamento hospitalar. Estes programas mostram maior eficácia quando iniciados no período pré-operatório e continuados no período pós-operatório.

Fica patente, na literatura considerada os domínios a considerar no desenvolvimento de programas de RR nos períodos pré e pós-operatórios, no entanto não existe consenso quanto aos exercícios a incluir, número de séries, repetições, intervalos e frequência dos mesmos. Atendendo à falta de consenso, o desenvolvimento do programa de RR tem de ser individualizado e assentar na avaliação da pessoa alvo de intervenção, centrando-se nas suas necessidades e objetivos, para promover a sua adesão e conduzir ao sucesso do programa.

Futuramente, estudos para determinar orientações clínicas mais precisas relativamente ao desenvolvimento de programas de $R R$ na fase perioperatória são necessários, nomeadamente adaptados à população portuguesa, visto que a bibliografia encontrada reporta a contextos estrangeiros.

\section{BIBLIOGRAFIA}

Ahmad, A. M. (2018). Essentials of physiotherapy after thoracic surgery: What physiotherapists need to know. A narrative review. Korean Journal of Thoracic and Cardiovascular Surgery, 5I(5), 293-307. https://doi.org/10.5090/kjtcs.2018.51.5.293

Ávila, A. C. de, \& Fenili, R. (2017). Incidência e fatores de complicações pulmonares pósoperatórias em pacientes submetidos à cirurgias de tórax e abdome. In Revista do Colegio Brasileiro de Cirurgioes (Vol. 44, Issue 3, pp. 284-292). https://doi.org/10.159o/oroo699120170030II

Boden, I., El-Ansary, D., Zalucki, N., Robertson, I. K., Browning, L., Skinner, E. H., \& Denehy, L. (2018). Physiotherapy education and training prior to upper abdominal surgery is memorable and has high treatment fidelity: a nested mixed-methods randomisedcontrolled study. Physiotherapy (United Kingdom), 104(2), 194-202. https://doi.org/Io.1016/j.physio.2017.08.008

Diário da República. (2019). Regulamento das Competências específicas do enfermeiro especialista em Enfermagem de Reabilitação. Diário Da Républica, $2^{a}$ Série - $n .^{\circ} 85-3$ de Maio de 2019, 13565-13568. https://dre.pt/home/-/dre/122216893/details/maximized 
Fujimoto, S., \& Nakayama, T. (2019). Effect of combination of pre- and postoperative pulmonary rehabilitation on onset of postoperative pneumonia: a retrospective cohort study based on data from the diagnosis procedure combination database in Japan. International Journal of Clinical Oncology, 24(2), 21I-221. https://doi.org/10.1007/sior47-0181343-y

Gao, S., Barello, S., Chen, L., Chen, C., Che, G., Cai, K., Crisci, R., D’Andrilli, A., Droghetti, A., Fu, X., Ferrari, P. A., Fernando, H. C., Ge, D., Graffigna, G., Huang, Y., Hu, J., Jiao, W., Jiang, G., Li, X., ... Ho, A. M. H. (2019). Clinical guidelines on perioperative management strategies for enhanced recovery after lung surgery. Translational Lung Cancer Research, 8(6), I174-1187. https://doi.org/10.21037/tlcr.2019.12.25

Ge, X., Wang, W., Hou, L., Yang, K., \& Fa, X. (2018). Inspiratory muscle training is associated with decreased postoperative pulmonary complications: Evidence from randomized trials. Journal of Thoracic and Cardiovascular Surgery, 156(3), 1290-1300.e5. https://doi.org/ro.1016/j.jtcvs.2018.02.105

Gil, A. C. (2010). Como Elaborar Projetos de Pesquisa (Atlas (ed.); $5^{\mathrm{a}}$ Edição).

Kabir, M. F., Jahan, S., Hossain, M. Z., Chakrovorty, S. K., Sarker, A. H., Hossain, M. A., Hossain, K. M. A., Kamal, S. M. M., \& Akter, S. (202I). Effect of Chest Physiotherapy along with Early Mobility after Abdominal Surgery. European Journal of Medical and Health Sciences, 3(I), 150-I56. https://doi.org/ro.24018/ejmed.2021.3.1.687

Kendall, F., Oliveira, J., Peleteiro, B., Pinho, P., \& Bastos, P. T. (2018). Inspiratory muscle 1307 training is effective to reduce postoperative pulmonary complications and length of hospital stay: a systematic review and meta-analysis. Disability and Rehabilitation, 40(8), 864-882. https://doi.org/10.1080/o9638288.2016.1277396

Liu, J., Wang, Y., Dui, J., \& Wang, G. (2020). Effects of respiratory rehabilitation nursing on improving postoperative respiratory function and quality of life of patients with lung cancer surgery. International Journal of Clinical and Experimental Medicine, 13(10), 7920-7927.

Lohiya, M. L., Malviya, A., Sharma, D. D., Ram, S., Chauhan, M. K., \& Chauhan, L. S. (2018). Prophylactic Chest Physiotherapy in Major Abdominal Surgery among Elderly Patients. Journal of the Indian Academy of Geriatrics, I4(2). https://doi.org/I0.35262/jiag.vi4i2.64-68

Lumb, A. B. (2019). Pre-operative respiratory optimisation: an expert review. Anaesthesia, 74, 43-48. https://doi.org/ro.III/anae.14508

Marseu, K., \& Slinger, P. (2017). Perioperative lung protection. Korean Journal of Anesthesiology, 7o(3), 239-244. https://doi.org/10.4097/kjae.2017.70.3.239

Miskovic, A., \& Lumb, A. B. (2017). Postoperative pulmonary complications. British Journal of Anaesthesia, II8(3), 317-334. https://doi.org/ı0.1093/bja/aexoo2

Odor, P. M., Bampoe, S., Gilhooly, D., Creagh-Brown, B., \& Ramani Moonesinghe, S. (2020). Perioperative interventions for prevention of postoperative pulmonary 
complications: Systematic review and meta-analysis. The BMJ, 368, 3-7. https://doi.org/1o.1136/bmj.m540

Ordem dos Enfermeiros. (2018). Reabilitação Respiratória - Guia Orientador de Boa Prática. https://www.ordemenfermeiros.pt/media/544I/gobp_reabilitação-

respiratória_mceer_final-para-divulgação-site.pdf

Schwartz, J., Parsey, D., Mundangepfupfu, T., Tsang, S., Pranaat, R., Wilson, J., \& Papadakos, P. (2020). Pre-operative patient optimization to prevent postoperative pulmonary complications - Insights and roles for the respiratory therapist: A narrative review. Canadian Journal of Respiratory Therapy, 56(December), 79-85. https://doi.org/ro.2939o/CJRT-2020-029

Spruit, M. A. (2014). Pulmonary rehabilitation. European Respiratory Review, 23(131), 55-63. https://doi.org/10.1183/09059180.00008013

Wang, Y. Q., Liu, X., Jia, Y., \& Xie, J. (2019). Impact of breathing exercises in subjects with lung cancer undergoing surgical resection: A systematic review and meta-analysis. Journal of Clinical Nursing, 28(5-6), 717-732. https://doi.org/Io.III/jocn.I4696

Zhou, K., Lai, Y., Wang, Y., Sun, X., Mo, C., Wang, J., Wu, Y., Li, J., Chang, S., \& Che, G. (2020). Comprehensive pulmonary rehabilitation is an effective way for better postoperative outcomes in surgical lung cancer patients with risk factors: A propensity score-matched retrospective cohort study. Cancer Management and Research, 12, 8903-8912. https://doi.org/ro.2147/CMAR.S267322 